\title{
DPGraph: A Benchmark Platform for Differentially Private Graph Analysis
}

\author{
Siyuan Xia \\ University of Waterloo \\ siyuan.xia@uwaterloo.ca \\ Yihan $\mathrm{He}$ \\ CIMS, New York University \\ yihan.he@nyu.edu
}

\author{
Beizhen Chang \\ University of Waterloo \\ beizhen.chang@uwaterloo.ca \\ Yuchao Tao
Duke University
uchao.tao@duke.edu
}

\author{
Karl Knopf \\ University of Waterloo \\ kknopf@uwaterloo.ca \\ $\mathrm{Xi} \mathrm{He}$ \\ University of Waterloo \\ xi.he@uwaterloo.ca
}

\begin{abstract}
Differential privacy has become an appealing choice for analyzing sensitive data while offering strong privacy protection, even for complex data types like graphs. Despite a decade of academic efforts in designing differentially private algorithms for graph analysis, few works have been used in practice. This is due to their complexity in the choice of privacy guarantees and parameter/environmental configurations, or due to their scalability issues for large datasets.

To bridge the gap between theory and practice, we present DPGraph, a web-based end-to-end benchmark platform built for researchers and practitioners to evaluate private algorithms on graph data. This platform supports a rich set of tunable algorithms for popular graph statistics, such as degree distribution and subgraph counting, with different differential privacy guarantees. A general framework for these algorithms has also been designed for users to tune the algorithms, by changing the sub-algorithms or re-distributing the privacy budget among the sub-algorithms. This enables users to understand the trade-off between privacy, accuracy, and performance of existing work and discover suitable algorithms for their applications.
\end{abstract}

\section{ACM Reference Format:}

Siyuan Xia, Beizhen Chang, Karl Knopf, Yihan He, Yuchao Tao, and Xi He. 2021. DPGraph: A Benchmark Platform for Differentially Private Graph Analysis. In Proceedings of the 2021 International Conference on Management of Data (SIGMOD '21), June 18-27, 2021, Virtual Event, China. ACM, New York, NY, USA, 5 pages. https://doi.org/10.1145/3448016.3452756

\section{INTRODUCTION}

Graph-like datasets such as email, social network, financial or health network, have the potential to help with improving these services or to improve scientific research. Frequently, these datasets are used for analysis of the relationships between the entities in the graph, and various statistics have been designed to address this. For example, graph data analytics have been used to improve digital contract tracing efforts [22].

Permission to make digital or hard copies of all or part of this work for personal or classroom use is granted without fee provided that copies are not made or distributed for profit or commercial advantage and that copies bear this notice and the full citation on the first page. Copyrights for components of this work owned by others than ACM must be honored. Abstracting with credit is permitted. To copy otherwise, or republish, to post on servers or to redistribute to lists, requires prior specific permission and/or a fee. Request permissions from permissions@acm.org.

SIGMOD '21, fune 18-27, 2021, Virtual Event, China

(c) 2021 Association for Computing Machinery.

ACM ISBN 978-1-4503-8343-1/21/06 . \$ \$15.00

https://doi.org/10.1145/3448016.3452756
However, privacy concerns fundamentally limit their potential application [1]. Traditional privacy-preserving practices for graphs are mainly based on the anonymization of the released graphs [5, 10, $14,27,34]$. However, these techniques usually fail to offer guarantees for multiple releases. For example, two $k$-anonymous releases can be combined to learn the sensitive locations of an individual exactly [9]. And the decisions made by anonymization algorithms can reveal information to the attacker, allowing them to breach privacy [32]. Strong privacy guarantees and tools are needed to support sensitive graph analysis.

Differential privacy (DP) [7] has emerged as a gold standard privacy guarantee for private data analysis and has been deployed by statistical organizations such as the US Census Bureau [21] and companies like Google and Microsoft. This privacy guarantee allows for multiple data releases and post-processing of the released information without breaking the privacy promise. However, the focus of the existing practical solutions has been on a flat table. A rich set of differentially private algorithms $[6,12,17,24,30]$ have been developed for graphs, but they are rarely used in practice due to the following challenges:

- Multiple variants of differential privacy are considered in the literature, such as node differential privacy $[6,17]$ and edge differential privacy $[12,24]$ to capture the rich structure of graph data. It is hard for non-privacy experts to choose the most appropriate privacy guarantee for their graph applications.

- Existing differentially private algorithms for graph analysis are often complex and have data-dependent errors. Their accuracy depends on the choice of the parameters, e.g., the maximum degree in the graph and the splitting of the privacy budget between different steps. The optimal algorithm that achieves the least error differs among graphs [6]. It is often challenging to identify the state-of-the-art algorithm for each graph statistic.

- Algorithms with strong utility promise cannot scale for largesized graphs if they are implemented naively. For example, to answer a degree distribution query on a graph with more than 300,000 nodes, one algorithm [6] takes half an hour while another [29] is $10-20$ times slower.

- Correctly implementing differentially private algorithms can be difficult $[20,23]$. Few algorithms have open source support. System environment supports also vary among algorithms. For example, graph-like data needs to be stored as relational databases $[15,18]$ while some algorithms use plaintext files for data input $[6,29,33]$. 
To address these challenges, we have built DPGraph, a web-based platform to benchmark differentially private algorithms for graph analysis. The goal of DPGraph is to provide an open-source and extendable framework for researchers and practitioners to evaluate existing algorithms for their privacy, accuracy, and efficiency, thus allowing them to discover the state-of-the-art for their applications. This framework also allows researchers to test their new algorithms against existing ones with ease. DPGraph has been inspired by prior work, DPComp [13], which benchmarks the accuracy of algorithms answering range queries over low dimensional tabular data. Unlike DPComp, which involves a single privacy guarantee and a single query type, DPGraph deals with multiple choices of privacy guarantees for graphs and multiple queries such as degree distribution and subgraph counting. In addition to evaluating the accuracy of the algorithms, DPGraph analyzes their run-time and scalability.

At the demonstration, the attendee will be shown the evaluation of an end-to-end differentially private algorithm that releases a graph statistic. Then, the attendee can interactively explore the trade-off space between privacy and accuracy of multiple algorithms on different datasets and privacy guarantees, and their runtime. The attendee can also evaluate new algorithms by modifying the default algorithm template from DPGraph.

\section{BACKGROUND}

Graphs considered in prior work of DP are mainly undirected and have no additional labels on nodes and edges. Given a graph $G=(V, E)$, where $V$ is the set of nodes and $E$ is a set of edges that connect pairs of nodes in $V$, prior work focus on releasing graph statistics, such as degree distribution [6], small subgraph counting [29], cut [11], graphons estimation [3], and generating synthetic graphs [28]. We summarize the key algorithms in Table 1 and organize them by privacy category and query type. We will first present the privacy guarantees for graphs and then highlight existing approaches from literature.

Variants of DP for graphs. The standard differential privacy for tabular data is defined as follows.

Definition 1 ( $\epsilon$-Differential Privacy [7, 8]). A randomized algorithm A satisfies $\epsilon$-differential privacy if for all pairs of neighbouring databases $D$ and $D^{\prime}$ and for any output set $S$, we have $\operatorname{Pr}[A(D) \in S] \leq e^{\epsilon} \times \operatorname{Pr}\left[A\left(D^{\prime}\right) \in S\right]$.

The output distribution of a differentially private algorithm is insensitive to small changes to the input database, where databases that only differ by the small change are called neighbours. For tabular data, the small change is defined as "differing by a row". In graphs, there are more options: (i) edge-DP considers pairs of graphs $G$ and $G^{\prime}$ differing by an edge; (ii) node-DP considers pairs of graphs differing by a node, where two nodes are different if they have different edges. We assume that the total number of nodes are fixed under node-DP. There are other variants, but we focus on these two notions and their corresponding algorithms in this demo.

If $G$ represents a social network, node-DP protects all the information of an individual while edge-DP protects only the relationships between persons. Here, node-DP offers stronger privacy protection than edge-DP. However, in most cases, queries can be answered more accurately under edge-DP than node-DP. We will

\begin{tabular}{|c|c|c|}
\hline DP Guarantee & Queries & Short Algo Name \\
\hline Edge/Node & $\operatorname{deg}$ & $\operatorname{degHis}\left({ }^{*}\right) ; \operatorname{degSeq}\left({ }^{*}\right)[12]$ \\
\hline Node & deg & $\begin{array}{l}\text { edgeAddHist; edgeAddCum; } \\
\text { edgeAddCumV [6] }\end{array}$ \\
\hline Node & deg, subG & nodeTrun; flowgraph $[17,29]$ \\
\hline Edge & subG & $\begin{array}{l}\text { smoothSens; noisyLocalSens } \\
\text { (for k-triangle only) [16] }\end{array}$ \\
\hline Edge/Node & subG & $\operatorname{recursive}(*) ; \operatorname{restricted}\left({ }^{*}\right)[2][4]$ \\
\hline Edge & subG & ladder [33] \\
\hline Edge & synG & wpinq $[25,26] ;$ ergmSyn $[16]$ \\
\hline Node & synG & erdosRenyiSyn [31] \\
\hline Local Edge & synG & ldpGen [28] \\
\hline
\end{tabular}

Table 1: DP algorithms for releasing degree distribution (deg) or/and releasing sub-graph counting (subG) or generating synthetic graphs (synG).

illustrate the utility difference using the Laplace mechanism [7] for degree distribution query $q$ that takes in $G$ and outputs a $|V|-$ dimensional vector of counts. To achieve $\epsilon$-edge DP (or node DP), the Laplace mechanism simply adds a noise vector drawn from the Laplace distribution with a mean 0 and standard deviation $\Delta(q) / \epsilon$, where $\Delta(q)$ is known as the global sensitivity of the query - the maximum change to the query answer (measured by the first norm of the vector) if changing an edge (or a node). For edge-DP, if adding or removing an edge, at most two nodes will change their degree values and thus four degree counts will be affected, i.e., $\Delta(q)=4$. For node-DP, changing a node can affect the degree values of $O(|V|)$ number of nodes. Hence, a larger amount of noise will have to be added to $q(G)$ to ensure node-DP than the noise for edge-DP.

Similar to degree distribution, many graph queries have a high sensitivity under node-DP. Even for edge-DP, some queries are highly sensitive to changes of edges, such as triangle counting. Dealing these queries has been the focus of DP algorithm design on graphs. We summarize the general approaches next.

The first approach transforms a given graph $G$ to a $\theta$-bounded graph $G^{\theta}$, where the maximum degree is bounded by $\theta$, so that the global sensitivity of the query on the new graph $G^{\theta}$ is also bounded. A smaller noise is then added to the query answer on $G^{\theta}$ to ensure DP. Several graph transformations have been proposed in prior work including node truncation [17, 18], edge removal [2], edge addition [6], and Lipschitz extensions [29]. The final noisy answer has two types of errors: the bias in the approximate query answer on $G^{\theta}$ due to graph transformation and the noise added directly to the query answer. When $\theta$ is large, few nodes or edges will be removed, but there will be more noise added. When $\theta$ is small, the noise will be small but $G^{\theta}$ will be too different from $G$. The optimal degree bound $\theta$ that gives the smallest error needs to be learned privately. Therefore, the optimal end-to-end algorithm differs among datasets even under the same privacy budget.

The second approach designs algorithms based on local sensitivity which is the maximum possible change for a given graph instance instead of considering all possible instances. As local sensitivity still leaks information of a graph, a smooth bound is computed for the local sensitivity of all possible databases at a distance from the given database distance $[4,15,17,24,33]$. The computation of a tight smooth bound for arbitrary queries $[4,17,24]$ is expensive or 


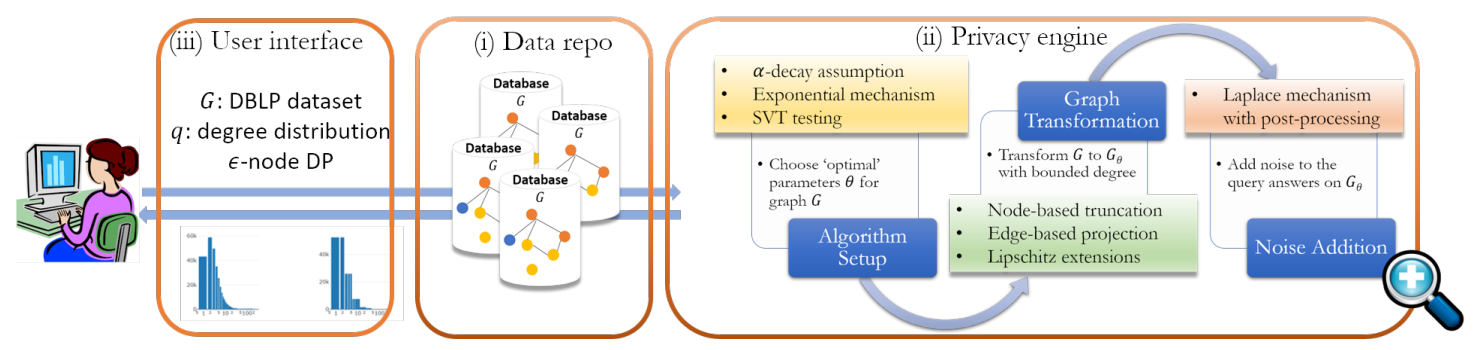

Figure 1: DPGraph Overview

requires specialization [33], especially for node-DP. A loose upper bound can be derived efficiently $[15,17]$, but may lead to large noise addition. Hence, the practicality of these algorithms will also be benchmarked in our system.

\section{OVERVIEW OF DPGRAPH}

DPGraph serves as a web-based platform to benchmark differentially private algorithms for graph analysis. This platform supports a large class of graph datasets and differentially private algorithms for graph queries. Data analysts or application developers who are privacy novices can use the system to understand the privacy, accuracy, and performance guarantees of existing algorithms with the optimal setting and choose a suitable algorithm for their applications. Privacy researchers are able to further understand the existing algorithms by tuning parameters and testing their new algorithms with ease.

DPGraph supports a large number of choices for privacy guarantees, datasets, queries, and algorithms. As shown in Figure 1, DPGraph involves three components: (i) a graph data repository with a multitude of public real and synthetic graph data,including social network data from Facebook and a DBLP collaboration network [19]; (ii) a back-end privacy engine which contains a suite of DP algorithms; (iii) a front-end user interface allowing users to interact with graph data repository and privacy engine. Users can select datasets, queries, and privacy guarantees via the interface. DPGraph will first list all possible algorithms that answer the chosen query and satisfy the chosen privacy guarantee, and then return a comparison of the accuracy and performance of these algorithms.

Privacy engine. The privacy engine has two modes. The first mode will present all end-to-end DP algorithms with default parameters. These algorithms are categorized by their privacy guarantees and query types. Their accuracy and run-time have been evaluated over a wide range of graph datasets and privacy budgets offline. These results can be explored by users directly with DPGraph interface. The second mode offers detailed exploration of all the global sensitivity-based mechanisms that are efficient. In this mode, users can download the default algorithm templates that are framed of several algorithmic modules, as shown in Figure 1: (i) algorithm setup module with the optimal configuration; (ii) graph transformation module, and (iii) noise addition module. Each module has more than one sub-algorithm. For example, the choice of optimal degree bounds can be based on $\alpha$-decay assumption [29], or learned privately using exponential mechanism [6] or sparse vector technique [18]. For high-dimensional query answers such as degree distribution or degree sequence, post-processing steps such as enforcing non-negativity counts and non-decreasing sequence for cumulative degree histograms play an important role in enhancing the accuracy of query answers. Composing different sub-algorithms from the three modules with different privacy budget allocations gives end-to-end algorithms of various accuracy levels.

User interface. DPGraph allows users to select datasets, queries, and choices of privacy guarantee via the user interface. Based on these choices, DPGraph displays a list of algorithms. As not all the algorithms have short run-time, especially on large datasets, the first prototype of DPGraph offers an offline mode. In this mode, the accuracy and the run-time of all algorithms have already been evaluated and saved. For each dataset and a privacy budget, we ran each algorithm multiple times. The noisy answer returned by the algorithm was compared with the true answer. We use the L1 norm as accuracy metrics for the degree distribution query and relative error for subgraph counting queries. Plots of both accuracy and run-time v.s. the total privacy budget will be displayed on the interface for comparisons. DPGraph also allows users to easily compare the performance of specific algorithms across multiple predefined privacy levels. By checking/un-checking an algorithm from the list of algorithms, users can hide or show this algorithm's result from the plot. This feature is particularly useful if users want to compare the performance of two specific algorithms against each other, or to remove traces of certain algorithms that are not of users' interest from the evaluation plot. For new algorithms modified from the default algorithm templates, DPGraph offers online mode for evaluation and comparison.

Limitations and Future Work. Future development of DPGraph will seek to address the limitations of the current prototype. First, we plan to add support for more complex aggregate graph queries,such as sets of graph queries instead of a single counting query. Second, we will ensure that the online execution of DPGraph is scalable for large datasets and is resilient to malicious uploading behavior.

\section{DEMONSTRATION SCENARIOS}

The attendee will be directed to the dashboard of DPGraph, which presents a set of benchmark datasets and queries for graphs. We will first show the attendee how to explore the accuracy and privacy trade-off for algorithms that satisfy node-DP and answer degree distribution query.

The demonstrator will first click the tab "Accuracy Frontier" from the dashboard and then select "Facebook" (one of the available datasets) and "Degree Distribution" (one of the queries). A 


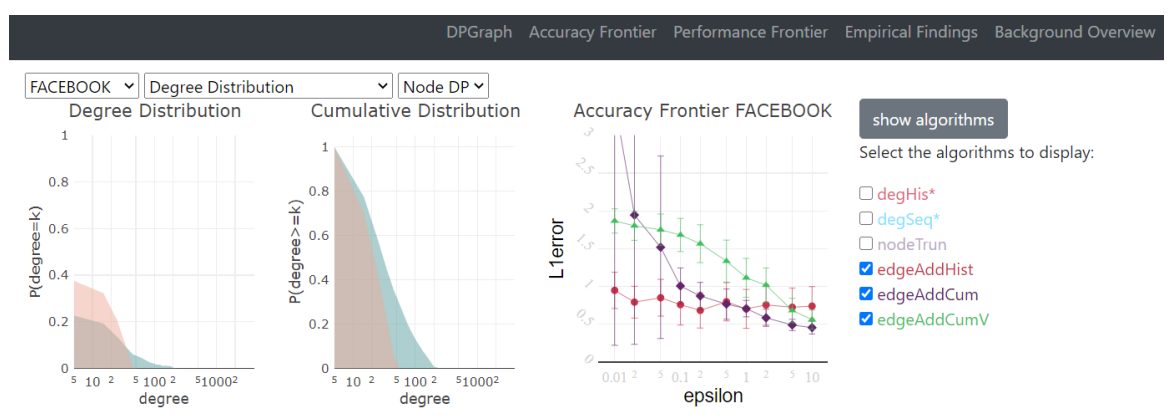

Figure 2: A screen shot of DPGraph visualizing the privacy-accuracy frontier, when selecting 'Node DP' algorithms for answering 'degree distribution' over 'Facebook' dataset. The blue and pink density plots show the true and noisy degree/cumulative distributions respectively. The error vs. epsilon plot shows the accuracy of the selected algorithms at different privacy budgets.

visualization of the true query answer for this dataset will then be displayed as the blue density plots shown in Figure 2. In particular, the blue plots on the left and in the middle show the true density degree distribution and the true cumulative degree distribution of the selected dataset, respectively. When the demonstrator selects "Node DP" (one of the DP guarantees), a list of available algorithms (degHis* [12], degSeq* [12], etc. from Table 1) for this combination of input choices will be shown. The trade-off plot of the accuracy of these algorithms and the privacy budget will be displayed (the third plot in Figure 2). The accuracy measure for degree distribution is measured as the L1 distance between the noisy degree distribution and the true degree distribution. Every algorithm is run 10 times for a given privacy budget $(\epsilon)$, and the mean and the standard deviation of the L1 errors vs. the privacy budget are reported in the plot.

Then, the demonstrator will let the attendee to select a subset of algorithms to inspect the accuracy-privacy tradeoff of the algorithms of their interest. Furthermore, the attendee can interact with the plots by clicking different points in the plots, and DPGraph will automatically generate a possible noisy output of the algorithm (the pink plots in Figure 2) so that the attendee can compare it with the true output of the given query. Since the noisy output is not the same every time, attendee can also click on the "Animate" button to examine the noisy density plots for different runs of the algorithm.

The privacy guarantee can also be weakened by switching from "Node DP" to "Edge DP". This change allows the attendee to see how the accuracy improves when switching to a weaker privacy guarantee. Besides the privacy guarantee, the attendee can choose another dataset of a different size. Plots for the same set of algorithms will be displayed to illustrate the different accuracy-privacy trade-off behavior of these algorithms on different datasets. The runtime and scalability evaluation of these algorithms will be shown on the "Performance Frontier" page. Similarly, in the tab for "Accuracy Frontier", the attendee can choose different sets of input combinations (privacy guarantee, query type, etc.) to inspect how these elements affect the runtime of the algorithms,

The demonstrators will also show attendees who are interested in the workflow of an algorithm and tuning the algorithm how to download the default algorithm and create new algorithms by adjusting the parameters or turning off/replacing some components of the sub-algorithms. These new algorithms can be then uploaded to DPGraph and be compared with the existing algorithms.

\section{REFERENCES}

[1] M. Barbaro and T. Z. Jr. A face is exposed for aol searcher no. 4417749. The New York Times, 2006.

[2] J. Blocki, A. Blum, A. Datta, and O. Sheffet. Differentially private data analysis of social networks via restricted sensitivity. In ITCS, 2013.

[3] C. Borgs, J. T. Chayes, A. D. Smith, and I. Zadik. Revealing network structure, confidentially: Improved rates for node-private graphon estimation. In FOCS, 2018.

[4] S. Chen and S. Zhou. Recursive mechanism: Towards node differential privacy and unrestricted joins. In SIFMOD, 2013.

[5] J. Cheng, A. W.-c. Fu, and J. Liu. K-isomorphism: Privacy preserving network publication against structural attacks. In SIGMOD, 2010

[6] W.-Y. Day, N. Li, and M. Lyu. Publishing graph degree distribution with node differential privacy. In SIGMOD, 2016.

[7] C. Dwork, F. McSherry, K. Nissim, and A. Smith. Calibrating noise to sensitivity in private data analysis. In TCC, 2006.

[8] C. Dwork, A. Roth, et al. The algorithmic foundations of differential privacy. Foundations and Trends ${ }^{\circledR}$ in Theoretical Computer Science, 2014.

[9] S. R. Ganta, S. P. Kasiviswanathan, and A. Smith. Composition attacks and auxiliary information in data privacy. In $K D D, 2008$.

[10] J. Gao, B. Song, Z. Chen, W. Ke, W. Ding, and X. Hu. Counter deanonymization query: $\mathrm{H}$-index based $\mathrm{k}$-anonymization privacy protection for social networks. In SIGIR, 2017.

[11] A. Gupta, A. Roth, and J. Ullman. Iterative constructions and private data release. TCC, 2012.

[12] M. Hay, C. Li, G. Miklau, and D. Jensen. Accurate estimation of the degree distribution of private networks. In ICDM, 2009.

[13] M. Hay, A. Machanavajjhala, G. Miklau, Y. Chen, D. Zhang, and G. Bissias. Exploring privacy-accuracy tradeoffs using dpcomp. In SIGMOD, 2016.

[14] M. Hay, G. Miklau, D. Jensen, D. Towsley, and P. Weis. Resisting structural re-identification in anonymized social networks. $V L D B, 2008$.

[15] N. Johnson, J. P. Near, and D. Song. Towards practical differential privacy for sql queries. $V L D B, 2018$

[16] V. Karwa, S. Raskhodnikova, A. Smith, and G. Yaroslavtsev. Private analysis of graph structure. ACM Trans. Database Syst., 39(3):22:1-22:33, Oct. 2014.

[17] S. P. Kasiviswanathan, K. Nissim, S. Raskhodnikova, and A. Smith. Analyzing graphs with node differential privacy. In TCC, 2013.

[18] I. Kotsogiannis, Y. Tao, X. He, M. Fanaeepour, A. Machanavajjhala, M. Hay, and G. Miklau. Privatesql: a differentially private sql query engine. VLDB, 2019.

[19] J. Leskovec and A. Krevl. SNAP Datasets: Stanford large network dataset collection. http://snap.stanford.edu/data, June 2014.

[20] M. Lyu, D. Su, and N. Li. Understanding the sparse vector technique for differential privacy. PVLDB, 2017.

[21] A. Machanavajjhala, D. Kifer, J. Abowd, J. Gehrke, and L. Vilhuber. Privacy: Theory meets practice on the map. In ICDE, 2008.

[22] Z. Mao, H. Yao, Q. Zou, W. Zhang, and Y. Dong. Digital contact tracing based on a graph database algorithm for emergency management during the covid-19 epidemic: Case study. 7MIR mHealth and uHealth, 9(1):e26836, 2021.

[23] I. Mironov. On significance of the least significant bits for differential privacy. In CCS, 2012.

[24] K. Nissim, S. Raskhodnikova, and A. Smith. Smooth sensitivity and sampling in private data analysis. In STOC, 2007.

[25] D. Proserpio, S. Goldberg, and F. McSherry. A workflow for differentially-private graph synthesis. In WOSN, 2012 
[26] D. Proserpio, S. Goldberg, and F. McSherry. Calibrating data to sensitivity in private data analysis: a platform for differentially-private analysis of weighted datasets. VLDB, 2014.

[27] K. P. Puttaswamy, A. Sala, and B. Y. Zhao. Starclique: Guaranteeing user privacy in social networks against intersection attacks. In CoNEXT, 2009.

[28] Z. Qin, T. Yu, Y. Yang, I. Khalil, X. Xiao, and K. Ren. Generating synthetic decentralized social graphs with local differential privacy. In CCS, 2017.

[29] S. Raskhodnikova and A. Smith. Lipschitz extensions for node-private graph statistics and the generalized exponential mechanism. In FOCS, 2016.
[30] V. Rastogi, M. Hay, G. Miklau, and D. Suciu. Relationship privacy: output perturbation for queries with joins. In PODS, 2009.

[31] J. Ullman and A. Sealfon. Efficiently estimating erdos-renyi graphs with node differential privacy. In Advances in Neural Information Processing Systems, 2019.

[32] R. C.-W. Wong, A. W.-C. Fu, K. Wang, and J. Pei. Minimality attack in privacy preserving data publishing. In $V L D B, 2007$.

[33] J. Zhang, G. Cormode, C. M. Procopiuc, D. Srivastava, and X. Xiao. Private release of graph statistics using ladder functions. In SIGMOD, 2015.

[34] E. Zheleva and L. Getoor. Preserving the privacy of sensitive relationships in graph data. In PinKDD, 2008. 